\title{
A Review on Mobile Cloud Computing
}

\author{
S M Shamim \\ Department of Information and \\ Communication Technology, \\ Mawlana Bhashani Science \\ and Technology University, \\ Santosh, Tangail, Bangladesh
}

\author{
Angona Sarker \\ Department of Information and \\ Communication Technology, \\ Mawlana Bhashani Science \\ and Technology University, \\ Santosh, Tangail, Bangladesh.
}

\author{
Ali Newaz Bahar \\ Department of Information and \\ Communication Technology, \\ Mawlana Bhashani Science \\ and Technology University, \\ Santosh, Tangail, Bangladesh
}

\section{Md. Atiqur Rahman \\ Department of Information and Communication Technology, Mawlana Bhashani Science and Technology University, Santosh, Tangail, Bangladesh.}

\begin{abstract}
Mobile cloud computing (MCC) is the availability of cloud computing services in a mobile environment. By providing optimal services for mobile users MCC incorporates the elements of mobile networks and cloud computing. In mobile cloud computing, all the data and complicated computing modules can be processed in clouds and mobile devices do not need a powerful configuration like CPU speed, memory capacity etc. However, the mobile devices are facing up with many struggles in their resources (e.g., battery life, storage, and bandwidth) and communications (e.g., privacy, mobility and security). These challenges have great affect in the improvement of service qualities. In this paper, we discuss the overview of mobile cloud computing technology together with the architecture, applications, major characteristics, security issues, advantage and limitation and possibly solution.
\end{abstract}

\section{Keywords}

Mobile Cloud Computing (MCC), Mobile environment, Mobile networks.

\section{INTRODUCTION}

Over the past few years, advances in the field of network based computing and applications mobile cloud computing (MCC) has been introduced as a potential technology for mobile services. It is the combination of mobile computing, cloud computing and wireless networks to bring high quality computational resources to network operators, mobile users, and cloud computing providers $[1,2]$. MCC is a new platform for combining the mobile devices and cloud computing to create a new infrastructure. It refers an infrastructure where both the data storage and the data processing happen outside of the mobile device [3]. In this architecture, cloud performs the heavy lifting of computing-intensive tasks and store large amounts of data. The rapid emergence of mobile computing (MC) [4] becomes a powerful trend in the development of information technology. However, the mobile devices in mobile computing are facing many problems in their resources (e.g., battery life, storage, and bandwidth) and communications (e.g., mobility and security) [5].

Due to major application model in the era of Internet, mobile cloud computing has become a significant research topic of the scientific and industrial communities. Its application is becoming more popular day by day. Therefore, different applications based on mobile cloud computing have been developed and served to users, such as Google's Maps ,Gmail and Navigation systems for Mobile, Voice Search, and various applications on an Android platform, MobileMe from
AppleandMotoBlur from Motorola. The increasing application of mobile computing is evident by the study of Juniper Research, which states that the consumer and enterprise market for cloud-based various mobile applications is $\$ 9.5$ billion [6]. The main objective behind the cloud computing is the delivery of different services, software and processing capacity over the Internet, increasing storage, reducing cost, automating systems and decoupling of service delivery from underlying technology, and providing flexibility and mobility of information in different purposes.

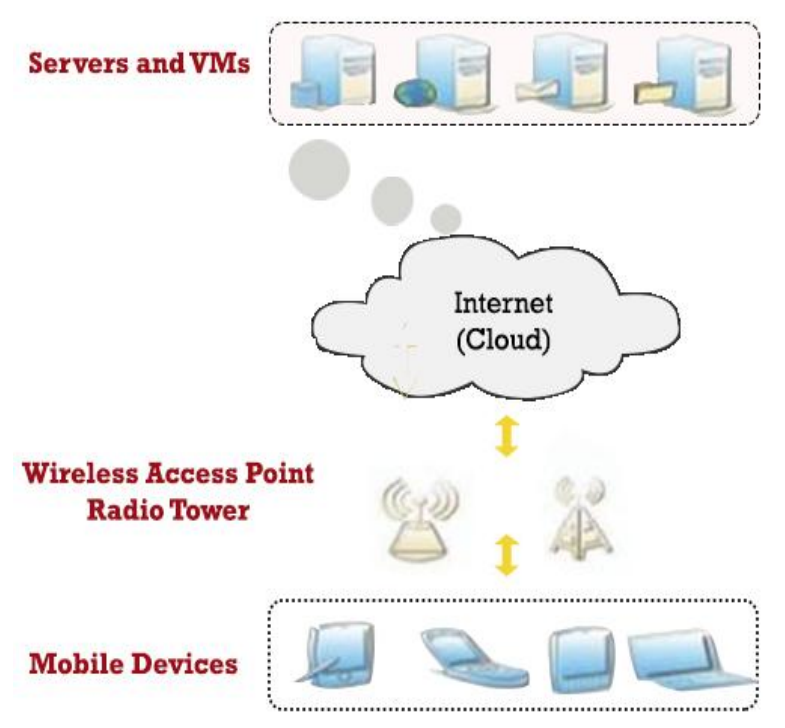

Fig-1: Mobile Cloud Computing

Mobile cloud applications move the computing power and data storage way from mobile phones and into the cloud. Aepona [7] describes mobile cloud computing as a new paradigm for mobile applications where data processing and storage are moved from mobile device to powerful and centralized computing platforms located in clouds over the internet. All these centralized applications are then accessed over the wireless connection based on a thin native client or web browser on the mobile devices. Alternatively, mobile cloud computing can be defined as a combination of mobile web and cloud computing $[8,9]$, which is the most popular tool for mobile users to access applications and services on the Internet. 


\section{MOBILE CLOUD COMPUTING ARCHITECTURE}

The general architecture of MCC can be shown in Figure 2. The main architecture of MCC is composed from the components: mobile users, mobile operators, internet service providers (ISP), cloud service providers, respectively [10].

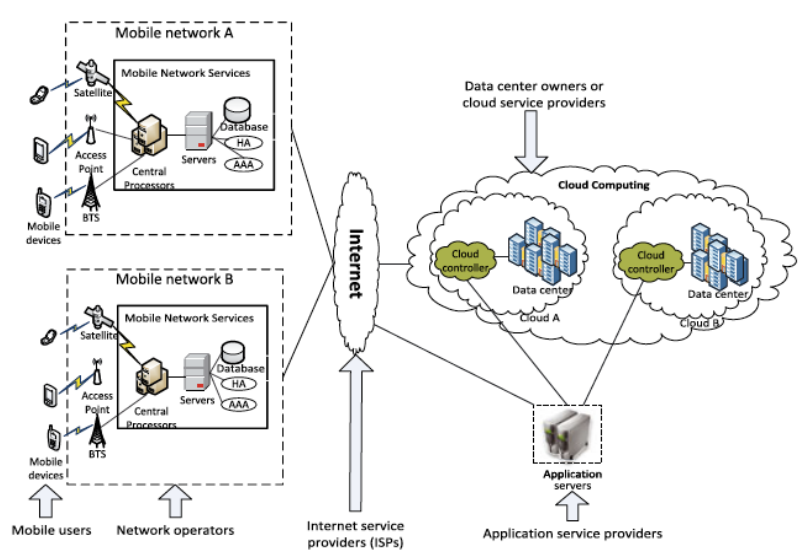

Fig-2: Architecture of Mobile Cloud Computing

The Mobile devices mainly mobile phones communicate with the mobile networks with the help of base stations, access points and/or satellite. The information's are transmitted from the mobile devices and these information's are operated on the central processors unit, servers and database on the mobile network provider side. Here, mobile network operators can provide valuable services to mobile phone users as an authorization, authentication and accounting based on the home agent and subscribers data stored in the databases. After that, the subscribers' requests to the cloud through the internet and cloud controllers process the requests to navigate to corresponding cloud services to provide mobile subscriber. The mobile cloud computing architecture provides effectiveness by using the advantages of the cloud computing. Four deployment models are identified for cloud architectures are:

\subsection{Private Cloud}

The cloud computing architecture is operated for a private organization and may be managed by the organization or a third party. It may be exist on premise or off premise.

\subsection{Community Cloud}

Various organization partake the cloud infrastructure which supports a particular community and it must have some communal concern such as security requisites, policy, vision and conformity considerations. Different organizations or third party may manage community cloud and it may be on physical server or may be hosted,

\subsection{Public Cloud}

The cloud infrastructure is made available to the general public or a large industry group. It may be owned by an organization selling cloud services.

\subsection{Hybrid Cloud}

The hybrid cloud architecture is a composition of two or more clouds that remain unique entities, but are bound together by standardized or proprietary technology.

The details explanations of cloud architecture could be different in different contexts. Therefore, four-layer architecture is explained in [11] to compare cloud computing with grid computing. [12] Described architecture for creating market-oriented clouds and [13] present architecture for webdelivered business services.

\section{APPLICATIONS OF MOBILE CLOUD COMPUTING}

Mobile cloud computing has a large number of application [14] in various fields and a wide range of potential mobile cloud applications have been recognized in the present literature. These applications are fall into different areas including natural language processing, image processing, sharing GPS, sharing Internet access, sensor data applications, crowd computing, querying and multimedia search.

\subsection{Image Processing}

In [14], the authors try to experiment with running GOCR and an optical character recognition (OCR) program on a collection of different mobile devices. A similar scenario is given in [15]. If user/subscriber visit foreign museum, he can't perceive the language written in each object of the museum. He can take picture of the object and using mobile cloud computing can understand the language written over the object.

\subsection{Natural Language Processing}

Language translation is one possible application for mobile cloud computing. Translation is a viable candidate for language processing since different sentences and paragraphs can be translated independently, and this is experimentally explored in [14] using Pangloss-Lite [16].

\subsection{Sharing GPS/Internet Data}

Through local-area or peer-to-peer networks data can be share among a group of mobile devices that are near each other. It is faster as well as cheaper [17].

\subsection{Sensor Data Applications}

Now-a-days almost every mobile devices are built with sensors which are used to read data. Some sensors such as GPS, accelerometer, thermo sensor, light sensor, clock and compass may be time stamped and associated with other phone readings. In order to gather precious information in different situation different queries can be executed.

\subsection{Multimedia Search}

Mobile phones may store different types of multimedia content such as videos, photos, and music. To illustrate, Shazam is a music identification service for mobile phones that searches for similar songs in a central database. In mobile cloud, the searching could be executed on the contents of nearby phones easily.

\subsection{Social Networking}

Since sharing different user content is a popular way and we can interact with friends on social networks such as Facebook.

\section{MOBILE CLOUD COMPUTING CHARACTERISTIC}

The major characteristics of mobile clouds computing are listed below:

\subsection{Flexibility/Elasticity}

Users can rapidly access provision computing resources without human interaction. User Capabilities can be rapidly and elastically provisioned, in some cases dynamically, to quickly scale out or up. 


\subsection{Scalability of Infrastructure}

In the physical servers new nodes can be added or dropped from the network with limited modifications to infrastructure set up and software. According to demand mobile cloud architecture can scale horizontally or vertically easily.

\subsection{Broad Network Access}

User capabilities and ability are available over the network and can be accessed through standard mechanisms that promote use by heterogeneous platforms like mobile phones, laptops, and PDAs etc.

\subsection{Location Independence}

Location independence is another characteristic of mobile cloud computing. There is a sense of different location independence where customer generally has no control or knowledge over the exact location of the provided resources. But it may be able to specify location at a higher level of abstraction from country, state, or datacenter.

\subsection{Reliability}

Through the use of multiple redundant site reliability can be improved and this makes cloud computing more worthy for disaster recovery applications and business continuity.

\subsection{Economies of Scale and Cost Effectiveness}

In order to take advantage of economies of scale mobile cloud implementations, regardless of the deployment model, tend to be as large as possible. Large number of mobile cloud deployments may be located close to cheap power stations and low-priced real estate, for lower costs.

\section{MOBILE CLOUD COMPUTING SECURITY}

In mobile cloud computing applications security and privacy are the key issues and still face some enormous challenges [18]. User's privacy and integrity of data or applications is one of the key issues in securing mobile cloud computing. It is the combination of cloud computing and mobile networks. For this the security related issues are then divided into two categories: cloud security and mobile network user's security; $[19,20,21,22]$.

\subsection{Mobile Network User's Security}

A large number of security vulnerabilities and threats such as malicious codes are known to the different mobile devices such as cellular phones, Smartphone's, PDAs, laptops etc. Some of the applications to these mobile devices can cause privacy issues for users [21]. There are the two major issues concerning the subscriber's security.

\subsubsection{Security for Mobile Applications}

The easiest ways to find security threats will be installing and running security software and antivirus programs on mobile devices. All the mobile devices are fixed with processing and power limitations. To protect devices from these threats could be more difficult compared to regular computers. To find threat detection and security mechanisms in the cloud several approaches have been developed. Before using a certain mobile application on mobile, it may go through some level of threat evaluation. First it will be verified and if malicious are not detect, file are sent to the user mobile devices. Mobile devices are performs only lightweight activities such as execution traces transmitted to cloud security servers instead of running anti-virus software or threat detection programs locally.

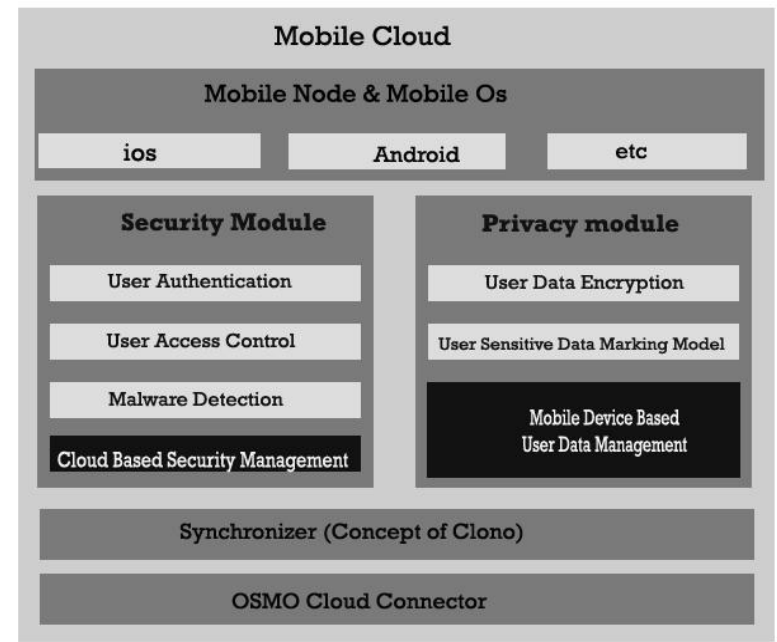

Fig-3: Mobile Cloud Computing Security Architecture Overview

\subsubsection{Privacy}

By providing private information including indicating current location and user's important information creates scenarios for privacy issues. To illustrate, the use of location based services (LBS) which are provided by global positioning system (GPS) devices. Various threats for exposing private information can be reduced by selecting and analyzing the enterprise needs and needs only specified services to be acquired and moved to the cloud network instead of running anti-virus software or threat detection programs locally.

\subsection{Securing Information on the Cloud}

For storing large amount of data or applications individuals and enterprises may take advantage over the cloud. However, integrity, authentication, and digital rights of data or application have to ensure during processing.

\subsubsection{Integrity}

The entire mobile cloud user must ensure the integrity of their information stored on the cloud network. All access must me authenticated and verified. In order to preserving integrity for one's information that is stored on the cloud is being proposed by giving different approaches. For example, entire information stored by each individual or enterprise in the cloud network is tagged or initialized to them wherein they are the only one to move, update or delete information.

\subsubsection{Authentication}

To secure the data access suitable for mobile environments using cloud computing large number of authentication mechanisms has been proposed. Some uses the open standards and supports the integration of deferent authentication methods. Such as illustrating the use of access or log-in IDs, password or PINS, authentication request etc.

\subsubsection{Digital Rights Management}

Piracy of various digital contents such as image, audio, video, and e-book programs becomes more and more popular day by day. A numerous solution has been proposed to protect these contents from illegal access are implemented such as provision of encryption and decryption keys to access these digital contents. Before accessing such digital contents on mobile devices coding or decoding platform must be done. 


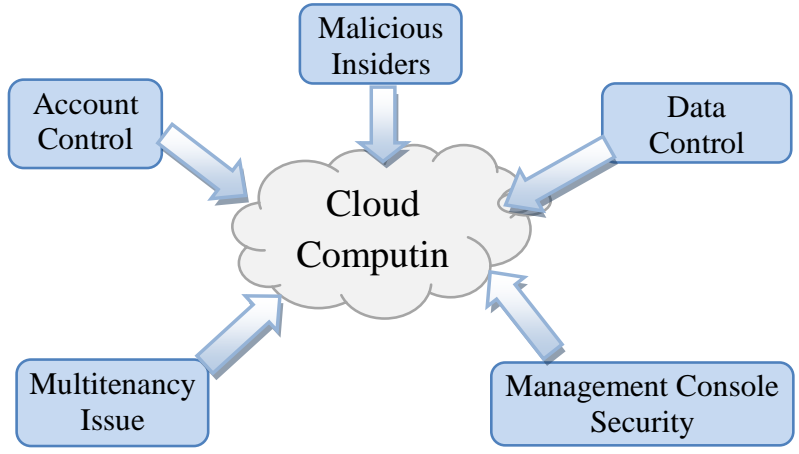

Fig-4: Categorization of threats

\section{MOBILE CLOUD COMPUTING ADVANTAGE}

There are many reasons including mobility, communication, and portability cloud computing is known to be a promising solution for mobile computing. Advantages of the consolidation of both mobile computing and cloud computing are combined by mobile cloud computing, thereby providing mobile user the optimal services [23]. These advantages are:

\subsection{Extending Battery Lifetime}

There are several solutions have been proposed to increase the CPU performance and organizes the disk and screen in an intelligent manner to reduce power consumption. In order to fulfill these solutions may require changes in the structure of mobile devices or require advance hardware that results in an increase of cost. But these changes may not be feasible for all mobile devices. In order to execute the large computations and complex processing from resource-limited devices like mobile devices to resourceful machines such as servers in clouds several computations offloading technique is proposed. Mobile cloud computing avoids taking a long application execution time on mobile devices which may results in large amount of power consumption.

\subsection{Improving Reliability}

By storing data or information on clouds is an effective way to increase the reliability whereby the data and application are stored and backed up on a number of computers. Hence the chance of data and application lost on mobile devices is reduced and ultimately reliability is increased. Moreover mobile cloud computing can be designed as a significance and compressive data security model for both service providers and users.

\subsection{Improving Data Storage Capacity and Processing Power}

Data storage capacity is also an important constraint for mobile devices. Mobile cloud computing is developed to enhance the mobile users to store/access the large data on the cloud network through wireless networks. There are several examples which are mostly used i.e. Amazon Simple Storage Service (Amazon S3) to provide file storage on the cloud network.

\subsection{Dynamic Provisioning}

Dynamic provisioning of resources is a flexible way for service providers and mobile users to run their various applications without advanced reservation of resources. Without storing data in mobile device it be stored in cloud and can be accessed dynamically.

\subsection{Scalability}

Due to flexible resource provisioning deployment of mobile applications can be performed. Internet Service providers can easily increase and expand an application and service without or with small constraint on the resource usage.

\subsection{Multi-tenancy}

Internet Service providers i.e. network operator or data center owner can share the resources and costs to provide variety of applications and for large number of users.

\subsection{Ease of Integration}

Multiple types of services from different service providers can be integrated easily through the cloud and Internet to meet the user's requirement.

\section{MOBILE CLOUD COMPUTING LIMITATION}

The main objective behind the mobile cloud computing is to provide a convenient and rapid method for subscriber to access and receive data from the cloud network [22]. Most of the challenge of mobile cloud computing comes from the characters of mobile devices and wireless networks and their own restriction and limitation. All these challenge makes application more complicated than on the fixed cloud devices. The entire limitations of mobile devices, quality of wireless communication and support from cloud computing to mobile are all important factors that affect accessing from cloud computing. Major limitation and solutions of mobile cloud computing is listed below:

\subsection{Low Bandwidth}

Since mobile network resource is much smaller compared with the traditional networks bandwidth is the one of major important issues in mobile cloud environment. Therefore, $\mathrm{P} 2 \mathrm{P}$ Media Streaming for distributing small bandwidth among the subscriber who are located nearby in the same area for the similar content such as the same video. Using this procedure, each user can transmits or exchanges parts of the same content with second users, which is resulted in improvement of content quality, especially for videos transmission.

\subsection{Security and Privacy in the Cloud}

In mobile cloud computing security and privacy has become the biggest concern. When establishing a remote cloud base infrastructure certainly any organization will give away private data and information which might be sensitive and confidential. Then it gives to the cloud service provider to manage, protect and retain them. The existence of the company might be jeopardous, so before taking any decision all the possible alternatives should be explored. Therefore, users might feel uncomfortable surrendering their data to a third party.

\subsection{Prone to Attack}

It is more vulnerable to external hack attacks and threats to store information in the cloud. Nothing on the internet is completely protected. Sensitive data and information may be stealth on the internet as many hackers and malicious users always lurk for the chances.

\subsection{Dependency and Vendor Lock-In}

One of the major disadvantages of mobile cloud computing is the implicit dependency on the internet service provider. It is really painful and cumbersome if one user wants to switch from one provider to some other provider as he has to transfer 
large number of data from the previous provider to the new one. This is another main reason why have to carefully and thoroughly contemplate in all options when picking a vendor.

\subsection{Limited Control and Flexibility}

Since all the applications and services run on remote or third party virtual environments, users have limited control over the whole function and execution of the hardware and software. In addition, since remote software is being used for mobile cloud computing, it usually lacks the features of an application running locally.

\subsection{Increased Vulnerability}

Privacy and security related cloud based solutions is more vulnerable target for hackers and malicious users as all cloud based solutions are exposed on the public internet. Many biggest players suffer from serious attack and security breakage in the internet. Nothing on the internet is fully secured.

The following strategies can be used to reduce to the challenges in mobile cloud computing:

For wireless connection upgrade bandwidth and make the web content more usable for mobile network using regional data centers.

* In order to reduce data delivery time deploy the application processing node at the 'edge' of cloud.

* Duplicate mobile devices to cloud using virtualization and image technologies, to process Data-Intensive Computing (DIC) and Energy-Intensive Computing, such as virus scanning in mobile devices.

* Optimize application push in cloud dynamically and the division with mobile terminals.

\section{CONCLUSION}

The major object behind the mobile cloud computing to empower the mobile user by providing a seamless and rich functionality, regardless of resource limitations of mobile devices. Though still now it is on the early stage of development, in future mobile cloud computing could become the major model for mobile application. According to a recent research, more than 240 million businesses will use cloud services through mobile devices by 2015 and will push the revenue of mobile cloud computing to $\$ 5.2$ billion. With this major importance, this paper has provided an overview of mobile cloud computing in which its definitions, architecture, and advantages have been presented. We have given an extensive review of current mobile cloud computing research in this paper. Therefore, highlighting the objective of mobile cloud computing, we have also described different definitions of mobile cloud computing in the literature. Our future work will focus on how security issue can be improved on mobile cloud computing.

\section{REFERENCES}

[1] Abolfazli, S., Sanaei, Z., Ahmed, E., Gani, A., \&Buyya, R. 2014. Cloud-based augmentation for mobile devices: motivation, taxonomies, and open challenges. Communications Surveys \& Tutorials, IEEE, 16(1), 337-368.

[2] Liu, F., Shu, P., Jin, H., Ding, L., Yu, J., Niu, D., \& Li, B. 2013. Gearing resource-poor mobile devices with powerful clouds: architectures, challenges, and applications. Wireless Communications, IEEE, 20(3), 14-22.

[3] Sarah Perez, August 4, 2009, Why cloud computing is the future of mobile, http://www.readwriteweb.com/archives/why_cloud_com puting_is_the_future_of_mobile.php, Retrieved on February 2015.

[4] Satyanarayanan M. 2010. Proceedings of the 1st ACM Workshop on Mobile Cloud Computing \& Services: Social Networks and Beyond (MCS).

[5] Tavel, P. 2007. Modeling and Simulation Design. AK Peters Ltd.

[6] S. Perez, Mobile cloud computing: $\$ 9.5$ billion by 2014 , http://exoplanet.eu/ catalog.php, 2010.

[7] White Paper. Mobile Cloud Computing Solution Brief. AEPONA, 2010.

[8] Christensen, J. H. 2009. Using RESTful web-services and cloud computing to create next generation mobile applications. In Proceedings of the 24th ACM SIGPLAN conference companion on Object oriented programming systems languages and applications (pp. 627-634). ACM.

[9] Liu, L., Moulic, R., \& Shea, D. 2010. Cloud service portal for mobile device management. In e-Business Engineering (ICEBE), 2010 IEEE 7th International Conference on (pp. 474-478). IEEE.

[10] Qian (Andy) Wang 2011. Mobile Cloud Computing, A Thesis Submitted to the College of Graduate Studies and Research In Partial Fulfillment of the Requirements, February 2011.

[11] Foster, I., Zhao, Y., Raicu, I., \& Lu, S. 2008. Cloud computing and grid computing 360-degree compared. In Grid Computing Environments Workshop, 2008. GCE'08 (pp. 1-10). IEEE.

[12] Buyya, R., Yeo, C. S., Venugopal, S., Broberg, J., \&Brandic, I. 2009. Cloud computing and emerging IT platforms: Vision, hype, and reality for delivering computing as the 5th utility. Future Generation computer systems, 25(6), 599-616.

[13] Huang, Y., Su, H., Sun, W., Zhang, J. M., Guo, C. J., Xu, J. M., \& Zhu, J. 2010. Framework for building a lowcost, scalable, and secured platform for Web-delivered business services. IBM Journal of Research and Development, 54(6), 4-1.

[14] Chang, J., Balan, R. K., \&Satyanarayanan, M. 2005. Exploiting rich mobile environments, 2005.

[15] Satyanarayanan, M. 2011. Mobile computing: the next decade. ACM SIGMOBILE Mobile Computing and Communications Review, 15(2), 2-10.

[16] Vallina-Rodriguez, N., \&Crowcroft, J. 2011. ErdOS: achieving energy savings in mobile OS. In Proceedings of the sixth international workshop on MobiArch (pp. 37-42). ACM.

[17] Marinelli, E. E. 2009. Hyrax: cloud computing on mobile devices using MapReduce (No. CMU-CS-09-164). Carnegie-mellonuniv Pittsburgh PA school of computer science, 2009. 
[18] Suo, H., Liu, Z., Wan, J., \& Zhou, K. 2013. Security and privacy in mobile cloud computing. In Wireless Communications and Mobile Computing Conference (IWCMC), 2013 9th International (pp. 655-659). IEEE.

[19] Huang, D., Zhou, Z., Xu, L., Xing, T., \&Zhong, Y. 2011. Secure data processing framework for mobile cloud computing. In Computer Communications Workshops (infocom wkshps), 2011 IEEE Conference on (pp. 614618). IEEE.

[20] Buyya, R., Broberg, J., \&Goscinski, A. M. (Eds.). 2010. Cloud computing: Principles and paradigms (Vol. 87). John Wiley \& Sons, 2010.
[21] Dinh, H. T., Lee, C., Niyato, D., \& Wang, P. 2013. A survey of mobile cloud computing: architecture, applications, and approaches. Wireless communications and mobile computing, 13(18), 1587-1611.

[22] Bahar, A. N., Habib, M. A., \& Islam, M. M. 2013. Security architecture for mobile cloud computing. International Journal, 3(3), 2305-1493.

[23] Kumar, L., Malik, N., Agghi, G., \&Anand, A. 2013. Mobile Cloud Computing. In International Conference on Computer Sciences and Applications. 